\title{
Impact on quality of life in peoples with painful diabetic peripheral neuropathy
}

\author{
Chong Hwa Kim¹, Su Jin Jeong ${ }^{1}$, Ji Oh Mok², Sung Rae Kim³, Ki Young Lee ${ }^{4}$
}

1. Division of Endocrinology and Metabolism, Department of Internal Medicine, Sejong General Hospital, Bucheon, Korea

2.Division of Endocrinology and Metabolism, Department of Internal Medicine, Soon Chun Hyang University Bucheon Hospital

3.Division of Endocrinology and Metabolism, Department of Internal Medicine, The Catholic University of Korea Bucheon ST.Mary's Hospital

4. Division of Endocrinology and Metabolism, Department of Internal Medicine, Gacheon University Gil Hospital

\section{Introduction}

Diabetic peripheral neuropathy (DPN) is the most common complication associated with diabetes. DPN can present as a loss of sensation, may lead to neuropathic ulcers, and is a leading cause of amputation.

DPN has serious detrimental effects on the physical, emotional, and social functioning of patients. Many DPN patients experience pain or discomfort, anxiety, depression, and limitations in activity, and may lose workdays or show decreased productivity as a result.

This study was performed to determine the impact on quality of life and sleep impairment of painful diabetic peripheral neuropathy(PDPN).

\section{Methods}

The study pool consisted of 200 randomly selected peoples with type 2 diabetic peripheral neuropathy. PDPN was diagnosed using visual analogue scales (VAS) and medical history. The patients were asked to answer the Brief Pain Inventory-Short Form (BPI-SF), Medical Outcomes Study Sleep (MOS-Sleep) Scale, EuroQol (EQ-5D), and VAS and estimate the quality of life in people with diabetic peripheral neuropathy.

A standardardized questionnaires such as MNSI, BPI-DPN, MOS 6 items sleep scale and EQ-5D were used to diagnosis and estimate the quality of life in people with diabetic peripheral neuropathy. A patient with diabetic peripheral neuropathy was defined as the patient who showed positive patients medical records or neuropathic symptoms(MNSI >2).

\section{Result}

Among the patients with diabetic peripheral neuropathy ( $n=200), 82(41 \%)$ were diagnosed with PDPN. PDPN was independently associated with age, fasting plasma glucose, hypertension, dyslipidemia and previous cerebrovascular events. All pain severity and interference measures were higher in patients with PDPN than those in patients with painless DPN and patients with PDPN reported more impaired sleep and lower EQ-5D and VAS scores.

Results: Pain score

Average and worst pain scores (BPI-DPN, 0-10 scales) were $4.6 \pm 2.4$ and $6.3 \pm 2.5$, respectively.

Pain substantially interfered ( $\geq 4$ on $0-10$ scales) with general activity, mood, walking ability, normal work, and relations with other people, sleep and enjoyment of life.
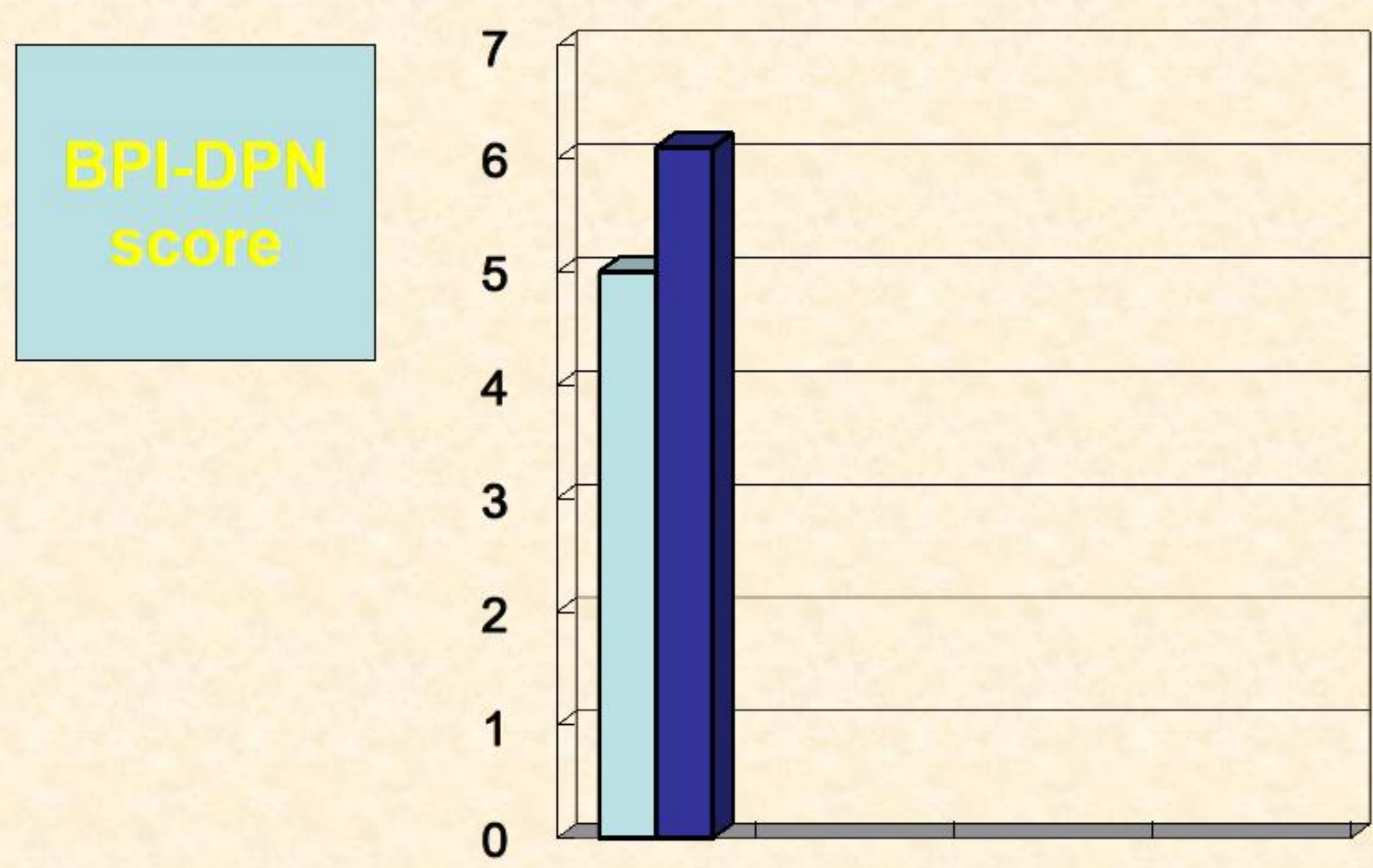

口average acore

$\nabla_{\text {worst score }}$

Patients With DPN Have Substantial Functional Impairment Across Multiple Domains

Impairment on modified Brief Pain Inventory score $\geq 4$

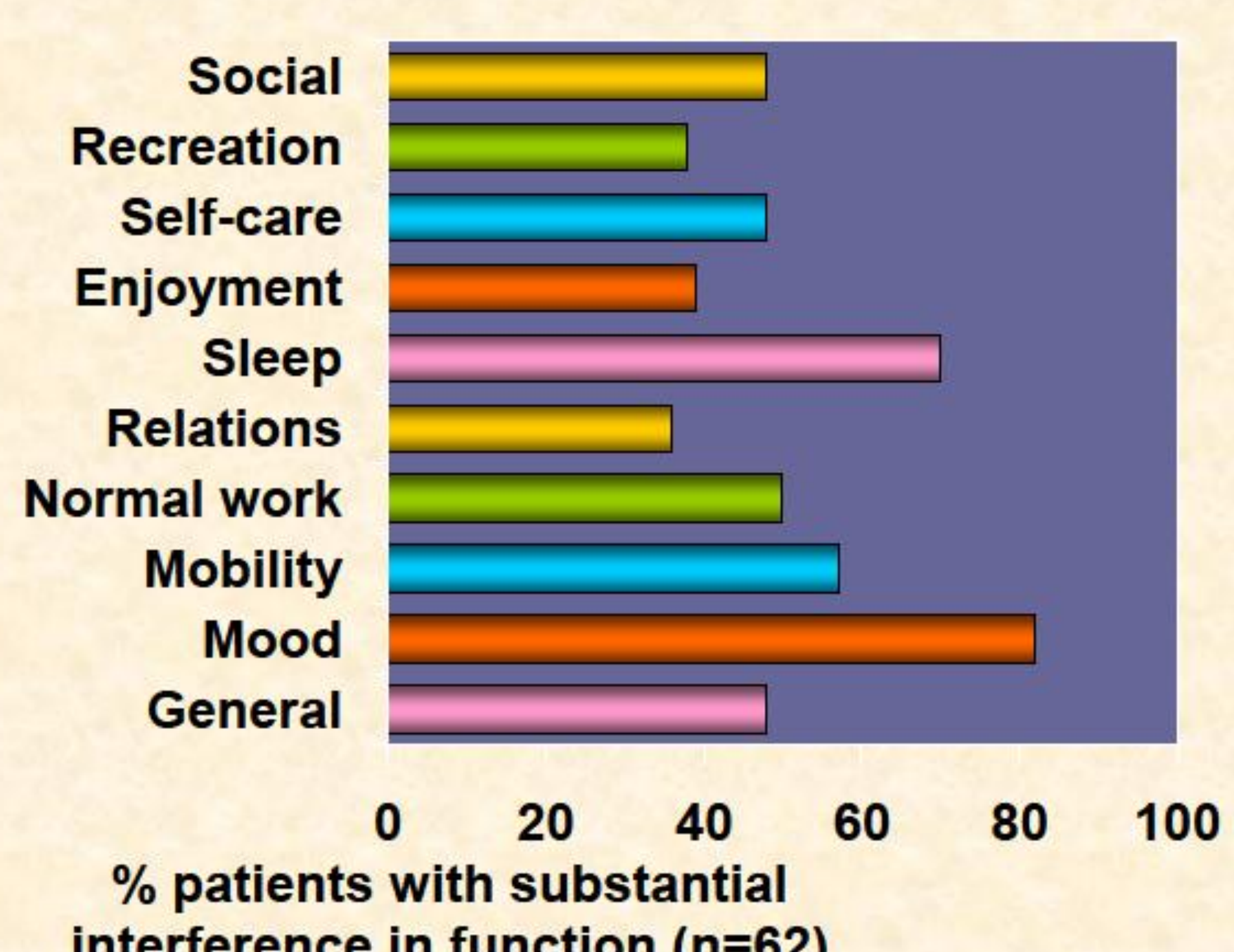

Medical Outcomes Study (MOS) 6-Item Sleep Scale*

Degree of sleep disturbance ${ }^{\star *}$

\begin{tabular}{|c|c|c|}
\hline Non-DPN (n=138) & $\square \mathrm{DPN}(\mathrm{n}=62)$ & $\begin{array}{c}(p<0.0001) \\
\text { Mean score }\end{array}$ \\
\hline Time to fall asleep & 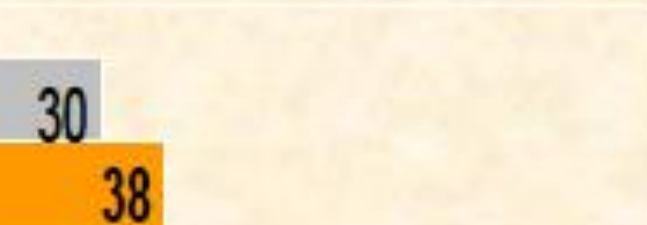 & $\begin{array}{l}30.38 \pm 32.72 \\
38.27 \pm 35.68\end{array}$ \\
\hline $\begin{array}{l}\text { Awakening short of breath or } \\
\text { with headache }\end{array}$ & $99^{97}$ & $\begin{array}{l}97.14 \pm 11.46 \\
94.01 \pm 17.04\end{array}$ \\
\hline Trouble falling asleep & $69^{80}-2-3-2$ & $\begin{array}{l}80.20 \pm 31.10 \\
69.36 \pm 36.89\end{array}$ \\
\hline Awakening during sleep & $69^{80}$ & $\begin{array}{l}80.43 \pm 29.58 \\
68.97 \pm 35.23\end{array}$ \\
\hline day & ${ }_{86}^{91}$ & $\begin{array}{l}91.12 \pm 20.35 \\
85.52 \pm 24.31 \\
\end{array}$ \\
\hline amount of sleep needed & ${ }_{33}^{26}$ & $\begin{array}{l}26.05 \pm 31.34 \\
32.69 \pm 35.00 \\
\end{array}$ \\
\hline
\end{tabular}

EuroQoL (EQ-5D)

Degree in influence of $\mathrm{QOL}^{*}$

【Non-DPN (n=138) \DPN(n=62)

Exercise

capability

Self -care

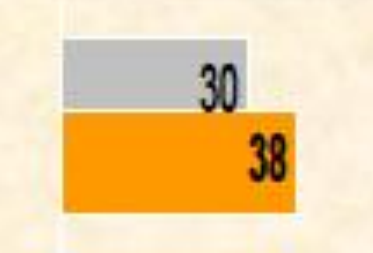

Daily activity

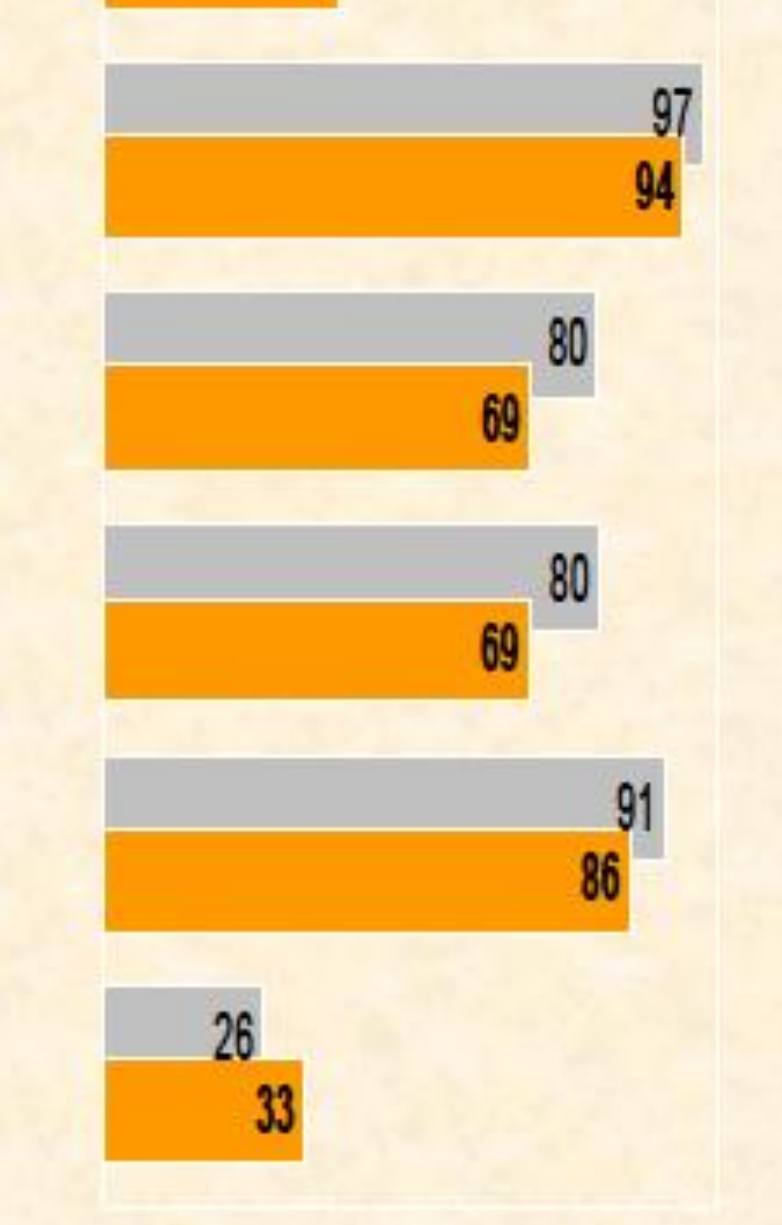

$(p<0.0001) \quad[$ Unit: \%]

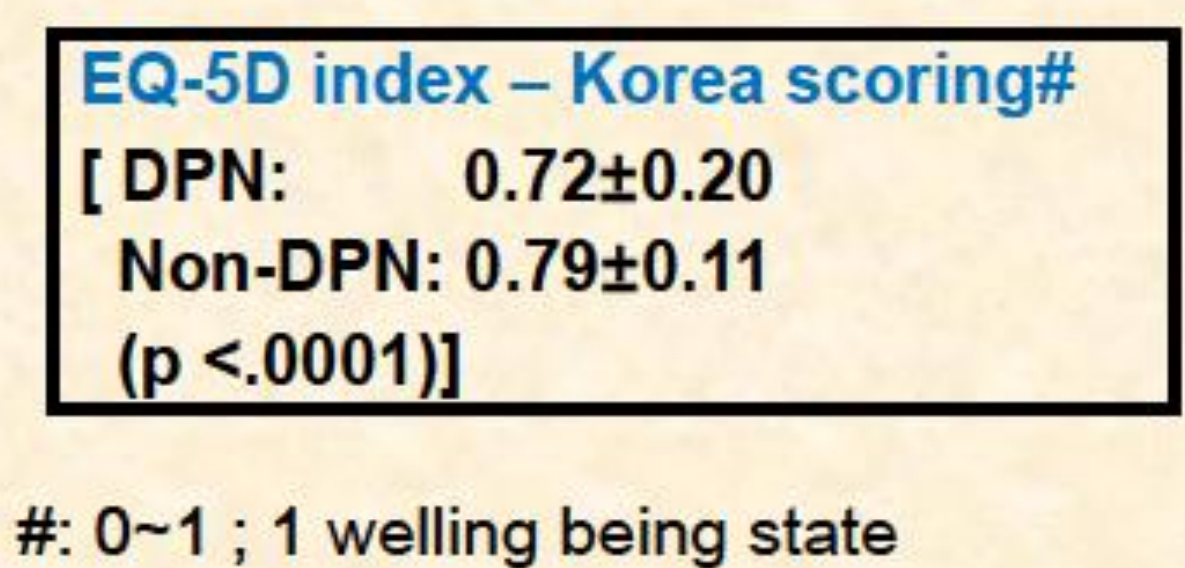

Pain

Idiscomfort

Anxiety

Idepression

${ }_{33}^{26}$

$* * 3$ score , 2, 3 rate of answer

200 patients had DPN and Pain severity index and pain interference items such as general activity, mood, walking, normal work, relationship, sleep and enjoyment of life in BPI-DPN were higher in patients with painful DPN compared to those in patients with painless DPN. Pain substantially interfered with mood, general activity, and caused decrements in many aspects of patients' lives; physical and emotional function, affective symptoms and sleep problems. MOS 6 items-sleep adequacy, respiratory problem during sleep, sleep initiation problem, sleep maintenance problem, and somnolence- sleep scale were lower in patient with painful DPN than painless DPN. EQ-5D index were lower in patients with painful DPN compared to those in patients with painless DPN.

\section{Conclusions}

Patients with painful DPN have greater discomfort during daily activities and sleep, and reduced QoL compared to patients with painless DPN. This study provide on the extent of the impact of pain on QoL in patients with painful DPN and physicians should carefully consider pain symptoms in patients with diabetic peripheral neuropathy

\section{References}

1. Jensen MP, Chodroff MJ, Dworkin RH. The impact of neuropathic pain on healthrelated quality of life: review and implications. Neurology. 2007;68:1178-1182 2. Gore M, Brandenburg NA, Hoffman DL, Tai KS, Stacey B. Burden of illness in painful diabetic peripheral neuropathy: the patients' perspectives. J Pain. 2006;7:892-900

3. Boulton AJ, Vileikyte L, Ragnarson-Tennvall G, Apelqvist J. The global burden of diabetic foot disease. Lancet. 2005;366:1719-1724. 